durch verdünnte reine Schwefelsäure in Sulfat verwandelt, dessen Gewicht innerhalb eines hinreichenden Temperaturintervalls von dem vollständigen Entweichen der überschüssigen Schwefelsäure bis zur beginnenden Rothglühhitze unverändert $2,0136 \mathrm{Grm}$. betrug. Das berechnete Gewicht, $\Psi n=55, s=32, \theta=16$ gesetzt, wäre 2,0111 gewesen.

Hiernach war ich der Ueberzeugung, dass dieses Manganoxydul genügend rein sei, um es zu den obengedachten Controlversuchen anwenden zu dürfen. Mit dem von Schneider $=54$ bestimmten Atomgewicht des Màngans würde freilich der zuletzt berichtete Versuch nicht in Einklang zu bringen sein.

Is erlohn, im Juni 1872.

Apparat zur Bestimmung von Gasen in Flüssigkeiten. Von

\title{
E. Reichardt.
}

Die bis jetzt vorgeschlagenen und in Anwendung gebrachten Apparate zur Bestimmung der in Wasser gelösten Gase sind meistentheils wenig handlich und hindern durch die ausgesuchte Complication namentlich die allgemeine Verwendung, ohne gerade eine grössere Genauigkeit zu versprechen. Erst im 9. Jahrg. dieser Zeitschr. S. 364 ist ein neuer Apparat zu diesem Zwecke von Herbert Mc. Leod beschrieben, der, sinnreich eingerichtet, dennoch an den oben berührten Mängeln gleichfalls leidet.

Der einfachste Apparat, welcher schliesslich noch am Meisten gebraucht wird, besteht in der mit dem zu untersuchenden Wasser gefüllten Flasche - Kochflasche u. s. w. - und die Gasleitungsröhre mündet direct unter Quecksilber in die Gasmessröhre. Bei dem Kochen wird natürlich Wasser übergetrieben, ersetzt das Quecksilber in der Gasmessröhre, tritt auch theilweise in die Quecksilberwanne über, so dass man die Gase stets über und mit Wasser in Berührung erhält und misst. Nach Beendigung des Versuches muss der Apparat sofort getrennt werden, um ein Uebersteigen von Quecksilber zu verhindern, ein Grund, den Versuch überhaupt früher zu beenden, und Sicherheit, dass alles Gas ausgetrieben, erhält man hierbei nicht. 
272 Reichardt: Apparat zur Bestimmung von Gasen in Flüssigkeiten.

Bei dem aufgefangenen Gase tritt nunmehr die Schwierigkeit ein, dasselbe genaner zu prüfen. - Ein Fehler - und der Versuch muss nochmals wiederholt werden.

Bunsen hat in den gasometrischen Methoden den von Regnault gegebenen Apparat zum Auffangen, Messen und Prüfen der Gase aufgenommen, and gewiss ist derselbe empfehlenswerth, jedoch einer allgemeinen Verwendung tritt die Complication und der Preis entgegen.

Der hier beschriebene Apparat zeichnet sich durch Einfachheit and äusserst leichte Handhabung aus, befindet sich auch nunmehr fast 2 Jahre ununterbrochen bei mir im Gebranche; die gewonnenen Resultate stimmen so gut überein, wie derartige Versuche sie überhaupt ergeben.

Zunächst habe ich bei dem Austreiben der Gase aus dem Wasser Quecksilber überhaupt umgangen, während die Messung sowohl, als Prüfung der Gasproben stets über Quecksilber ausgeführt werden; an und für sich ist es bei den bisher gebräuchlichen Methoden entweder unmöglich, ohne Wasser das Gas zu messen, da ersteres stets mit übergetrieben wird, oder man beendet den Versuch zu früh.

Fig. 14.

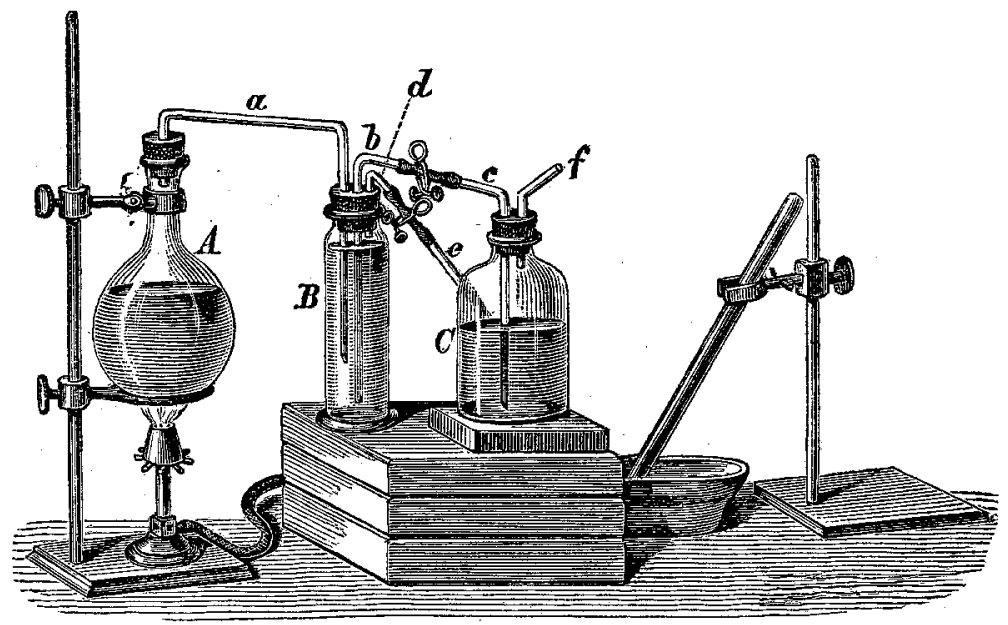

Flasche A enthält das zu untersuchende Wasser, sie fasst 1 Liter oder 100 Cubikcentimeter, je nach Bedürniss, oder kann auch unmittelbar durch die betreffende Flasche Wasser - Sodawasser und dergl. - ersetzt werden; in letzterem Falle wird die Verbindung mit dem weiteren Apparate nur durch die bekannten Hähne mit Schraube und Wechselhahn herge- 
gestellt. Der Haltbarkeit der Flaschen wegen geschieht dann das Erwärmen auch im Paraffinbade. Für gewöhnlich genügt eine gewöhnliche, vollständig angefüllte Kochflasche, deren Inhalt bestimmt wurde.

Flasche B dient als Gasometer und ist ein schmales, cylinderförmiges Gefäss mit 3fach durchbohrtem Stopfen versehen. In der einen Durchbohrung befindet sich Röhre a, deren längerer Schenkel in Flasche $\mathrm{B}$ bis etwa zu $2 / 3$ der Höhe derselben eingeht, der andere sehr kurze Schenkel endet in einem Kautschukstopfen, welcher auf Flasche A passen muss. Die zweite Durchbohrung enthält die nur rechtwinkelig gebogene Röhre $b$, deren längerer Theil bis fast auf den Boden von $B$ reicht, der kürzere, äussere Theil steht durch Kautschukrohr und Quetschhahn mit Röbre $\mathrm{c}$ in Verbindung. In der dritten Durchbohrung befindet sich ein kurzes Stück gebogenes Glasrohr d, welches genan mit dem Stopfen in Flasche B abschneidet, der äussere Theil steht durch Kautschukrohr und Quetschhahn mit der wie gewöhnlich geformten Röhre e in Verbindung, weiche die Gase in die nebengezeichnete Quecksilberwanne und unter die Gasmessröhre führt.

Flasche $\mathrm{C}$ von ca. $1 / 2-1$ Liter Inbalt hat einen 2 fach durchbohrten Stopfen; in der einen Durchbohrung befindet sich ein winkelig gebogenes Glasrohr c, dessen längerer Theil fast bis zum Boden von Flasche $\mathrm{C}$ reicht, der kürzere Schenkel ist durch Kautschukrohr und Quetschhahn mit Flasche B, durch Rohr b, in Verbindung gesetzt. Die zweite Durchbohrung enthält ein kurzes umgebogenes Glasrohr f, dessen eines Ende unten mit dem Stopfen abschneidet; das äussere dient, wie die Handhabung des Apparates ergeben wird, dazu, um Druck durch Einblasen auf das innen befindliche Wasser auszuüben.

Bei dem Gebrauche wird Flasche A vollständig mit dem za untersuchenden Wasser angefüllt, $\mathrm{B}$ und $\mathrm{C}$ werden mit ausgekochtem und noch warmem, in geschlossenen Gefässen fast erkaltetem, destillirtem Wasser so gefüllt, dass $\mathrm{C}$ etwa nur zur Hälfte Wasser behält, wie die Figur deutlich angibt. Indem man nun bei f einbläst, füllt man sehr leicht Flasche $B$ nebst den Röhren a und d-e mit Wasser an; ist der Zweck bei $d-e$ völlig erreicht, so schliesst man den zwischen $d$ und e befindlichen Quetschhahn und ebenso endlich den zwischen $b$ und $\mathrm{c}$ befindlichen, wodurch auch Röhre a voll Wasser er- und gehalten wird. Nunmehr verschliesst man Flasche A mit dem Korke der Röhre a, der dadurch entstehende Druck wird leicht durch die Kautschukröhren, welche bei b-c 
274 Reichardt: Apparat zur Bestimmung von Gasen in Flüssigkeiten.

und $d-e$ sind, ausgeglichen, sodann öffnet man Quetschhahn $b-c$ und beginnt A zu erwärmen.

Die nunmehr ausgetriebenen Gase gehen durch a nach B, damit sie nicht durch $\mathrm{b}-\mathrm{c}$ nach $\mathrm{C}$ mit entweichen können, ist Röbre a nicht so tief in B eingesenkt; die Gase sammeln sich in B, das als Gasometer dient, an, will man sie messen oder sonst prüfen, so öffnet man $d-e$ und bläst bei $f$, wodurch sie unter die Gasmessröhre getrieben werden.

Das Aufsammeln des Gases in $\mathbf{B}$ and Uebertreiben in die Gasmessröhre kann jederzeit und in beliebigen Portionen geschehen, so dass zu verschiedenen Prüfungen eben so viele Theile verwendet werden können, deren Gasmengen allerdings einzeln zu messen and dann zusammen $\mathrm{zu}$ zählen sind.

Mittlerweile wird das Wasser in A immer wärmer, geräth endlich in lebhaftes Kochen, wodurch mehr und mehr davon übergetrieben wird, die Gase sammeln sich in $\mathrm{B}$, das Wasser circulirt ungehindert in $\mathrm{B}$ u. $\mathrm{C}$; sobald Abkühlung eintritt, das Feuer entfernt wird, füllt sich $A$ von selbst durch Zurücksteigen des Wassers wieder an und zeigt deutlich, ob noch Gasreste verbleiben oder der Versuch völlig beendet ist. Zuletzt wird das Wasser in B auch kochend heiss, so dass etwa gebundenes Gas frei werden müsste.

Der Versuch kann jeden Augenblick unterbrochen oder ohne Aufhören bis zur Beendigung fortgesetzt werden, und bei einiger Aufmerksamkeit kann man in der Gasmessröhre das Gas allein auffangen, indem man vorsichtig das die Leitungsröhre füllende Wasser herausbläst und dann erst das Gas in die Röhre leitet. Die vollständige Entfernung der durch Kochen auszutreibenden Gase kennzeichnet gewöhnlich ein starkes Aufstossen der Flüssigkeit.

Die Versuche können rasch auf einander folgen und sind äusserst einfach in der Ausführung. Ueber die Genauigkeit derselben mögen einige Beispiele entscheiden, welche keineswegs besonders ausgewählt wurden.

Wasser der Röhrenleitung von Jena wurde direct derselben entnommen und ergab in 1 Liter an Gas:

22. Januar 1870.

I. 50,02 CC. II. $49,11 \mathrm{CC}$.

Das Gas bestand in 100 Vol.-Theilen aus: 
I.

Sauerstoff . . . . . . 15,31

Stickstoff . . . . . . . 35,32

Kohlensäure. . . : . . . . 49,37
II.

15,78

37,67

46,55

Das Verhältniss von $0: \mathrm{N}$ ist bei I. wie $1: 2,30$, bei II. wie $1: 2,38$. Dasselbe Wasser wurde am 30 . Januar 1870 untersucht und ergab im Liter:
I. 42,48 II. 43,59 CC. Gas.

Das Gas bestand in 100 Vol.-Theilen aus:

I.

Sauerstoff · . . . 14,14

Stickstoff . . . . . 37,83

Kohlensäure * . . . 48,03
II.

13,16

39,23

47,61

Die Differenzen beruhen hier theilweise auf der Kohlensäure, welche durch Zersetzung der Salze allmählich frei wird.

Wasser, aus dem Wasserkasten geschöpft, ergab in Liter folgende Gasmengen:
I. 53,20
II. $54,97 \mathrm{CC}$.

Das Gas bestand aus:

I.

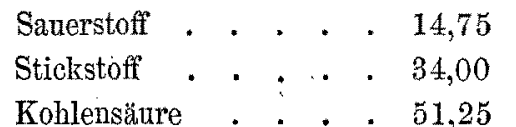

Kohlensäure . . . . 51,25

Die Versuche sind stets parallel za gleicher Zeit angestellt worden, um eben Controle augenblicklich zu haben und stimmen gewiss so gut, wie es bei derartigen Verhältnissen nur zu erwarten ist.

Ueber nentralen phosphorsauren Kalk, Darstellung und Löslichkeit desselben.

Vor

\section{E. Reichardt.}

Um die Löslichkeit des phosphorsauren Kalkes in kohlensaurem Wasser zu beweisen, ist als Collegienversuch folgendes Experiment zu empfehlen : 